\title{
A Variação dos Indicadores Básicos do Índice de Desenvolvilmento Humano dos Países Latino- Americanos no Período de 2007-2010: uma Decomposição com uso da Metodologia Shift- Share
}

Variation of Basic Indicators Index of Human Desenvolvilmento Latin American Countries in the Period 2007-2010: a decomposition using the Shift-Share Methodology

Bruno Theylon Oliveira Dias ${ }^{7}$

Gilson Batista de Oliveira

\section{Resumo}

Este artigo apresenta a variação dos indicadores saúde, educação e renda, componentes básicos do Índice de Desenvolvimento Humano (IDH), do qual, utilizarmos a América Latina como região e os países que a compõe como locais, constatou-se nos países latino-americanos que alguns desses indicadores sofreram variação, provocados pela crise econômica que afetou o mundo em maior e menor grau de intensidade. Para isso, utilizou-se o método shift-share que ao decompor os elementos do IDH, pode-se constatar variações em seus componentes, dessa forma, subdivide-se esse países em quadrantes de acordo com o grau de variação que esses países sofreram entre 2007 e 2010. Espera-se, esclarece que apesar de a crise ter afetado a América Latina pela via econômica principalmente no que tange a exportação de commodities, isso implica afetar outros setores sociais, no caso, saúde, educação e renda.

Palavras-chave: Crise econômica, variação de IDH, decomposição, América Latina, shift-share.

\section{Abstract}

This article presents the variation of health indicators, education and income, basic components of the Human Development Index (HDI), which, we use Latin America as a region and the countries that compose and local, was found in Latin American countries that some of these indicators were

\footnotetext{
${ }^{7}$ Bruno Theylon Oliveira Dias: Graduando do curso de Relações Internacioanis e Integração, pela Universidade Federal da Integração Latino-Americana (UNILA). Bolsista do PROBIC/UNILA. E-mail: bruno.dias@unila.edu.br

Gilson Batista de Oliveira: Doutor em Desenvolvimento Econômico - UFPR. Professor da Universidade Federal da Integração Latino-Americana - UNILA. E-mail: gilson.oliveira@unila.edu.br
} 
variation, caused by the economic crisis that affected the world in greater and lesser intensity. This was done using the method shift-share elements that decompose the HDI, one can see variations in their components, thus it is divided into quadrants countries in accordance with the degree of variation between these countries suffer 2007 and 2010. It is hoped, explained that although the crisis has affected Latin America through economic especially regarding the export of commodities, this implies affect other social sectors, in the case, health, education and income.

Keywords: economic crisis, variation in HDI, decomposition, Latin America, shift-share.

\section{Introdução}

A América Latina tem em seu histórico diversas etapas de desenvolvimento que na maioria das vezes, esses países não conseguiram unificar crescimento econômico e social para que pudesse gerar uma economia sustentável. Entender as dinâmicas econômicas e sociais desses países através da decomposição do Índice de Desenvolvimento Humano (IDH) pelo método shift-share, através dos dados dos anos de 2007 e 2010, é uma forma de compreender um pouco mais da dinânica da relação economia e sociedade.

A América Latina é muito ampla e pode gerar dificuldade de compreensão em sua totalidade. Nesse trabalho, optou-se pela definição do IMEA (2009) que classifica 21 países como formadores da América Latina, são eles: Argentina, Belize, Bolívia, Brasil, Chile, Colômbia, Costa Rica, Cuba, Equador, El Salvador, Guatemala, Haiti, Honduras, México, Nicarágua, Panamá, Paraguai, Peru, República Dominicana, Uruguai e Venezuela. Entretanto, dado a carência de dados básicos, alguns desses países não puderam ser incluídos na decomposição.

Com esta pesquisa, de forma geral, verificam-se os impactos da crise financeira mundial de 2008 nos indicadores básicos do IDH. Especificamente, estuda-se os diferentes níveis de desenvolvimento que se encontram os países que compõe a América Latina, através do método shift-share restringindo-se apenas na decomposição dos indicadores do IDH, no período de 2007 - 2010 .

A crise econômica de 2008 que causou um mal estar financeiro mundial a partir da quebra do banco Lehman Brothers nos EUA e logo se espalhou principalmente para países europeus e atingiu as mais diversas partes do mundo, chega aos dias de hoje com incertezas de até quando essa crise perdurará.

Na América Latina, observou-se que essa crise sistêmica não atingiu com tanta força, apesar de ter baixado o preço de commodities, além de gerar fuga massiva de capitais especulativos, também houve uma considerável diminuição do ritmo de investimentos externos em um primeiro momento.

Para driblar a crise, governos fazem cortes e readaptações de diversos setores da política econômica de um país, isso implica em um impacto 
direto na qualidade de vida da sociedade, ou seja, no Índice de Desenvolvimento Humano.

A pesquisa usou informações divulgadas nos relatórios sobre o desenvolvimento humano publicados pelo Programa das Nações Unidas para o Desenvolvimento - PNUD de 2007 e 2010.

Após a aplicação da metodologia shift-share, identificou-se quais os indicadores básicos mais expressivos na variação do IDH no período de 2007 - 2010, por conseguinte, depreende-se quais foram os indicadores básicos mais afetados pela crise financeira mundial de 2008 .

\title{
O Índice de Desenvolvimento Humano
}

O Índice de Desenvolvimento Humano (IDH), é publicado no Relatório de Desenvolvimento Humano $(\mathrm{RDH})$, lançado pela primeira vez num período de transformações do sistema político e econômico global, em 1990, tem como objetivo de usar outros parâmetros além do econômico, para mensurar o nível de desenvolvimento.

$\mathrm{O}$ IDH foi concebido pelo economista paquistanês Mahbub UI Haq em colaboração do economista indiano, nobel de 1998, Amartya Sem. O índice tornou-se o referencial de desenvolvimento em praticamente todos os países membros da Organização das Nações Unidas (ONU), que mantem o Programa das Nações Unidas para o Desenvolvimento (PNUD), órgão responsável pelo $\mathrm{RDH}$, (PNUD, 2009).

Segundo PNUD (2010), para rivalizar com o PIB, o IDH foi concebido de uma forma simples, apesar da avalanche de tabelas relacionadas. O IDH precisou ser idealizado de uma forma explicativa, baseado na longevidade, rendimento per capita e educação. Como afirma o PNUD Brasil:

\begin{abstract}
Além de computar o PIB per capita, depois de corrigi-lo pelo poder de compra da moeda de cada país, o IDH também leva em conta dois outros componentes: a longevidade e a educação. Para aferir a longevidade, o indicador utiliza números de expectativa de vida ao nascer. O item educação é avaliado pelo índice de analfabetismo e pela taxa de matrícula em todos os níveis de ensino. A renda é mensurada pelo PIB per capita, em dólar PPC (paridade do poder de compra, que elimina as diferenças de custo de vida entre os países). Essas três dimensões têm a mesma importância no índice, que varia de zero a um. Disponível em: <http://www.pnud.org.br/idh/> Acesso em 10 de março de 2012.
\end{abstract}

A classificação do nível de desenvolvimento dos países, segundo PNUD (2009), funciona em uma escala numérica que vai de 0 à 1 , onde quanto mais próximo de 1 , melhor é o nível de desenvolvimento. A classificação dos países baseado no nível do IDH funciona da seguinte forma:

i) IDH baixo: entre 0 e 0,499, aponta baixo desenvolvimento humano;

ii) IDH médio: entre 0,500 e 0,799, denota níveis de médio desenvolvimento humano; 
iii) IDH elevado: entre 0,800 e 0,899 , retrata altos níveis de desenvolvimento humano;

iv) IDH muito elevado: todos aqueles acima de 0,900, indica níveis de desenvolvimento humano muito elevados, do comparado aos demais.

Segundo PNUD (2010), apesar de o sistema de verificação do IDH ter sido lançado em 1990, boa parte dos países recalcularam seu IDH até 1975, proporcionando assim um melhor parâmetro de desenvolvimento nos quase 40 anos de verificação. O que se constatou nesses últimos anos, é que a média mundial do IDH obteve um crescimento considerável, chegando a atingir um crescimento de cerca de $41 \%$ entre 1975 e 2010, sendo que apenas três países por motivos de conflitos obtiveram em 2010 um IDH menor do que em 1970, são eles República Democrática do Congo, Zâmbia e Zimbabwe.

A partir de 2010, quando se completou 20 anos de $\mathrm{RDH}$, foram acrescentados outros elementos na aferição do IDH, além dos itens básicos como saúde; educação e renda, agora é contabilizado também outros aspectos como a distribuição do bem estar em termos de desigualdade de gênero e pobreza. O quadro a seguir retirado do PNUD 2010, resume um pouco dos três principais elementos:

\begin{tabular}{|c|c|}
\hline & Dimensões \\
\hline Saúde & $\begin{array}{l}\text { É determinado pela esperança de vida ao nascer, ou } \\
\text { seja, o número de anos que espera-se que um recém- } \\
\text { nascido venha a viver. Essa expectativa de vida é } \\
\text { determinada pela média de mortalidade. }\end{array}$ \\
\hline Educação & $\begin{array}{l}\text { A componente Educação é determinada pela média de anos de } \\
\text { escolaridade e anos de escolaridade esperado, onde o primeiro } \\
\text { com peso de } 2 / 3 \text { no cálculo geral, é referente a alfabetização, } \\
\text { geralmente concluída aos } 15 \text { anos e o segundo com peso de } 1 / 2 \\
\text { refere-se a taxa de matrícula em qualquer nível de educação. }\end{array}$ \\
\hline Renda & $\begin{array}{l}\text { O rendimento nacional bruto per capita, é medido em dólar pela } \\
\text { Paridade do Poder de Compra (PPC) e tem o objetivo de verifica } \\
\text { se o poder aquisitivo de uma pessoa consegue suprir suas } \\
\text { necessidades básicas, dessa forma determinando os padrões de } \\
\text { vida. }\end{array}$ \\
\hline
\end{tabular}

Quadro 1: Componentes do idh

Fonte: PNUD, 2010

O cálculo das dimensões do IDH é medido através dos valores que variam entre 0 e 1 , ficando da seguinte forma:

$$
\text { Índice de dimensão }=\frac{\text { valor efetivo-valor mínimo }}{\text { valor máximo -valor mínimo }}
$$


No cálculo dos índices (saúde, educação e renda) utiliza-se valores mínimos e máximos obtidos dentre os participantes do grupo de referência. Obtidos os índices básicos, o IDH é calculado através da média geométrica.

De acordo com o PNUD (2010), espera-se que o IDH seja capaz de apontar o quanto uma sociedade está desenvolvendo ao longo do tempo, como as capacidades humanas estão, de uma forma que seja possível identificar as opções que as pessoas tenham de assegurar sua própria subsistência através de escolhas participativas no sistema social.

$\mathrm{O}$ RDH de 2010 evidencia que, a diferença de desenvolvimento humano entre países ricos e países pobres tem diminuído consideravelmente nos últimos 20 anos. O índice porém, não é capaz de explicar as razões de tais diferenças na América Latina, também pode-se vislumbrar esse cenário de redução de desigualdade.

Dessa forma, ao fazer um recorte temporal para verificar o comportamento do IDH na América Latina entre os anos de 2007 e 2010, pode nos oferecer um mínimo de informações necessárias para constatar tais mudanças.

\title{
A Metodologia de Análise Shift - Share
}

O método shift-share é uma forma analise dos elementos que compõe um dado estatístico em duas escalas de tempo. Essa metodologia é bastante aplicada para verificar desenvolvimento econômico regional ou setorial.

\begin{abstract}
A analise de componentes de variação (ou shift-share) decompõe o crescimento de uma dada variável, medida a nível regional, em factores distintos que possam influenciar o seu comportamento...A ideia base é muito simples: as diferenças de crescimento em uma região podem ser atribuídas não só a diferenças relativas à composição produtiva de cada região...mas também, pode ser justificadas por diferentes vantagens de natureza locacional. (COSTA, 2002 p.803)
\end{abstract}

Na metodologia shift-share os elementos que compõe uma estrutura são estudados individualmente, de forma que possam ser relacionados com o todo, destacando como foi seu desenvolvimento ao longo do tempo e como tal desempenho pode influenciar no conjunto.

A utilização do shift-share vai além da visão analítica, passa para o campo das projeções ou planejamentos, uma vez que possibilita identificar quais e quando foram as mudanças mais impactantes no setor ou indicador analisado. Nesse método, as componentes de alteração diferencial ou variação diferencial devem ser isoladas afim de possibilitar uma comparação estatística. Por alteração diferencial, entende-se a possibilidade de um setor da economia ou indicador de desenvolver-se como o nome já diz - diferente de outro setor econômico ou indicador usado como parâmetro. "Mede a alteração naquele crescimento que resulta a influencia exercida por certos factores como vantagens de localização ou 
grau de competitividade no crescimento mais rápido ou mais lento de alguns dos sectores urbanos". ( VASCONCELLOS, 1984. p.222)

No entanto, como afirma Vasconcelos (1984) e Oliveira (2010), o shiftshare só é viável se os resultados obtidos de um certo estudo, puderem ser comparados com outro semelhante, para que daí se possa tirar conclusões sobre concentração econômica, especialização regional, dentre outros, dependendo qual for o objetivo do uso do método.

\begin{abstract}
A maioria dos modelos de análise de componentes de variação apresentam-se como identidades matemáticas, expressando a evolução de uma dada variável económica como função de três factores principais: o efeito do crescimento nacional(componente nacional), 0 efeito da composição sectorial da região (componente estrutural) e, ainda, o efeito de outros factores específicos da região (componente regional, concorrencial ou diferencial). (COSTA, 2002, p.804)
\end{abstract}

Após identificado e isolados os elementos regionais à nível de comparação com o nacional, o shift-share trabalha usando os resultados obtidos em uma expressão padrão, para fazer essa relação entre crescimento ou não do setor regional e diferenciações que levam a esse resultado em relação ao nível nacional. Nesse estudo, adaptado para os componentes do Índice de Desenvolvimento Humano, considerar-se-á nível nacional o total dos países da América Latina e como regional, cada país individualmente.

De acordo com Costa (2002) e Oliveira (2010), o modelo clássico da metodologia shift-share pode ser descrito da seguinte forma:

$$
\Sigma=\Delta X_{k} i k=\sum[X i k(t)-X i k(t-1)]_{k}=\sum[N X i k+S X i k+R X i k]
$$

$\Delta$ Xik é a variação observada na variável Xik;

Xik(t) representa a variável econômica $X$, na região i, no setor k e no momento t;

Nxik representa a componente nacional

Sxik representa a componente setorial ou estrutural

Rxik representa a componente regional, concorrencial ou diferencial.

Essas três ultimas variáveis, componente nacional, estrutural e diferencial, são obtidas da seguinte forma:

$$
\text { NXik }=g N X \times X_{i k}(t-1)
$$

Onde gNX é a variação percentual da variável X observada no nível nacional relativamente ao ano base $\mathrm{t}-1$; 


$$
\text { SXik }=(g N X k-g N X) \times X_{i k}(t-1)
$$

gNXk é a variação percentual da variável X observada a nível nacional, referente ao setor $\mathrm{k}$;

$$
\text { RXik }=(g i k-g N X K) \times X_{i k}(t-1)
$$

gik é a variação percentual da variável $X$, observada na região i no setor $\mathrm{k}$.

As componentes de variação podem nos indicar o crescimento ou não do objeto de estudo.

Para Haddad e Andrade (1989 apud Oliveira, 2010, p.122), "a análise shift-share permite a identificação do crescimento, entenda-se variação dos indicadores selecionados através da VLT - Variação Liquida Total, que é o crescimento observado menos o teórico(...)"

Partindo dos trabalhos de Oliveira (2010) e Simões (2005), pode-se sintetizar os resultados da aplicação da metodologia shift-share e classificar as regiões de acordo com as variações obtidas, conforme quadro 2.

\begin{tabular}{|c|c|c|c|}
\hline VLT & VLE & VLD & Tipo de País \\
\hline \multirow{3}{*}{+} & + & + & A1 \\
\cline { 2 - 4 } & + & - & A2 \\
\cline { 2 - 4 } & - & + & A3 \\
\hline \multirow{3}{*}{-} & - & + & B1 \\
\cline { 2 - 4 } & + & - & B2 \\
\cline { 2 - 4 } & - & - & B3 \\
\hline
\end{tabular}

\section{Quadro 2: Classificação e tipologia de variações}

Fonte: Adaptação de Simões (2005) e Oliveira (2010)

VLT a Variação Liquida Total positiva (+), indica aquelas localidades onde o crescimento locacional é maior ou igual ao crescimento regional (A1, A2 e A3) ou desde que a VLD supra as percas de VLE, de acordo com os parâmetros utilizados. Já VLT negativo (-) aponta todas aquelas localidades que tiveram crescimento menor que o regional (B1, B2 e B3) ou desde que o fator diferencial não seja suficientemente satisfatório.

$\mathrm{O}$ fato de A2 ter VLT positivo e B2 VLT negativo é porque o crescimento estrutural de B2 não é grande o bastante em relação ao regional de acordo com o elemento diferencial escolhido. Pode-se usar 0 mesmo exemplo para A3 e B1 de forma invertida, onde o fator locacional de A3 é grande o bastante para suprir VLE negativo, e B1 apesar de também ter VLD positivo, esse não consegue ser grande o bastante em relação ao fator estrutural. (SIMÕES, 2005; OLIVEIRA, 2010)

A adaptação do método para decomposição do Índice de Desenvolvimento Humano

$\mathrm{Na}$ atualidade, o método shift-share é aplicado amplamente nas mais diversas formas de análise regional, não só em relação aos indicadores de 
desenvolvimento econômico, mas nos chamados indicadores sociais, no nosso caso, nos componentes do Índice de Desenvolvimento Humano.

Conforme Oliveira (2010) é possível aplicar o método shift-share para decompor o IDH e com os resultados obtidos, pode-se verificar e classificar quais dos indicadores básicos (saúde, renda ou educação) mais influenciaram na variação do índice. De forma resumida, com base na tipologia do quadro 2, de referencia, pode-se classificar os países de acordo com o desempenho de cada indicador básico, quadro 3.

\begin{tabular}{|c|c|c|c|c|}
\hline \multirow[t]{2}{*}{ Descrição } & \multicolumn{3}{|c|}{$\begin{array}{l}\text { Resultados Possíveis para cada } \\
\text { Indicador Básico e para cada } \\
\text { Índice Selecionado }\end{array}$} & \multirow[b]{2}{*}{ Países } \\
\hline & $\begin{array}{c}\mathrm{VLT} \\
(\mathrm{N}-\mathrm{NX})^{*}\end{array}$ & $\begin{array}{l}\mathrm{VLE} \\
(\mathrm{SX})^{\star *}\end{array}$ & $\begin{array}{l}\text { VLD } \\
(\mathrm{RX})^{\star \star *}\end{array}$ & \\
\hline $\begin{array}{l}\text { Indicador de Educação (IE) } \\
\text { Indicador de Longevidade (IL) } \\
\text { Indicador de Renda (IR) }\end{array}$ & $\begin{array}{l}+ \\
+ \\
+ \\
- \\
- \\
-\end{array}$ & $\begin{array}{l}+ \\
+ \\
- \\
- \\
+ \\
-\end{array}$ & $\begin{array}{l}+ \\
- \\
+ \\
+ \\
- \\
-\end{array}$ & $\begin{array}{l}\text { A1 } \\
\text { A2 } \\
\text { A3 } \\
\text { B1 } \\
\text { B2 } \\
\text { B3 }\end{array}$ \\
\hline $\begin{array}{l}\text { Índice de Desenvolvimento } \\
\text { Humano (IDH) }\end{array}$ & $\begin{array}{l}+ \\
+ \\
+ \\
- \\
- \\
-\end{array}$ & $\begin{array}{l}+ \\
+ \\
- \\
- \\
+ \\
-\end{array}$ & $\begin{array}{l}+ \\
- \\
+ \\
+ \\
- \\
-\end{array}$ & $\begin{array}{l}\text { A1 } \\
\text { A2 } \\
\text { A3 } \\
\text { B1 } \\
\text { B2 } \\
\text { B3 }\end{array}$ \\
\hline
\end{tabular}

Quadro 3: Esquema para guiar a interpretação dos resultados da adaptação da metodologia shift-share para os indicadores básicos do índice de desenvolvimento humano Fonte: Oliveira, 2010.

De posse dos resultados, conforme Oliveira (2010), pode-se, ainda, construir gráficos indicativos de como anda o IDH de uma localidade, colocando os países em quadrantes que retratem de forma sintética a realidade de cada um. Para o autor, os elementos Eficiência Alocativa e Ativação Social, descritos no quadro 4, são os indicativos principais de como a administração pública aplicou os recursos para garantir ou não a qualidade do IDH. 


\begin{tabular}{|c|c|c|c|c|c|c|c|}
\hline \multirow{2}{*}{ Quadrante } & \multirow{2}{*}{ Países } & \multirow{2}{*}{$\begin{array}{l}\text { Eficiência } \\
\text { Alocativa }\end{array}$} & \multirow{2}{*}{$\begin{array}{c}\text { Ativação } \\
\text { social }\end{array}$} & \multirow{2}{*}{ VLT } & \multicolumn{2}{|c|}{ Componente } & \multirow{2}{*}{ Interpretação } \\
\hline & & & & & VLE & VLD & \\
\hline I & $\mathrm{A} 1$ & Presente & Presente & + & + & + & $\begin{array}{l}\text { íses com maior capacidade de ativação social, cuja } \\
\text { p teve mais sucesso na transformação do impulso de } \\
\text { crescimento em desenvolvimento, ou seja, na } \\
\text { internacionalização dos efeitos do processo de } \\
\text { scimento. Nesse quadrante devem figurar os países } \\
\text { pm as maiores variações positivas dos indicadores } \\
\text { ásicos componentes de cada índice estudado, que } \\
\text { ota maiores níveis de variação positiva da qualidade } \\
\text { vida, do desenvolvimento humano. Aqui, as nações } \\
\text { ssuem eficiência alocativa (VLS>0) e capacidade de } \\
\text { ativação social (VLD>0). }\end{array}$ \\
\hline \multirow{2}{*}{ II } & A3 & Ausente & Presente & + & - & + & $\begin{array}{c}\text { Países com capacidade e ativação social } \\
\text { intermediária, cuja ação consegue trazer resultados } \\
\text { individuais de cada indicador básico acima da média } \\
\text { da região, significando avanço na transformação do } \\
\text { impulso de crescimento em desenvolvimento. Em } \\
\text { resumo, a capacidade de ativação social (VLD>0) } \\
\text { consegue suplantar a ineficiência alocativa (VLE<0) e } \\
\text { ter um crescimento maior que a média da América } \\
\text { Latina (VLT>0). }\end{array}$ \\
\hline & B1 & Ausente & Presente & - & - & + & $\begin{array}{l}\text { Embora com crescimento total abaixo da média } \\
\text { regional (VLT negativo), significa perda líquida em } \\
\text { comparação com o universo da América Latina, os } \\
\text { países com esses resultados também possuem } \\
\text { capacidade de ativação social intermediária (boa), } \\
\text { pois a VLD positiva mostra que o índice estudado } \\
\text { teve um desempenho superior ao dos demais } \\
\text { países da região, porém, não conseguem superar a } \\
\text { ineficiência alocativa (VLE }<0) \text {. }\end{array}$ \\
\hline III & B3 & Ausente & Ausente & - & - & - & $\begin{array}{l}\text { Países com capacidade de ativação social fraca, } \\
\text { que denota fragilidade na internalização dos efeitos } \\
\text { gerados pelo processo de crescimento. Nesses } \\
\text { países, a sociedade local teve menos sucesso na } \\
\text { transformação do impulso de crescimento em } \\
\text { desenvolvimento, pois detêm as piores variações } \\
\text { dos indicadores básicos componentes de cada } \\
\text { índice estudado na região analisada, cuja falta de } \\
\text { ativação social é piorada pela ineficiência alocativa. }\end{array}$ \\
\hline \multirow[t]{2}{*}{ IV } & B2 & $\begin{array}{c}\text { Present } \\
\mathrm{e}\end{array}$ & Ausente & - & + & - & $\begin{array}{c}\text { Países com desempenho regular, que tende a } \\
\text { fragilidade, isto é, nações cuja sociedade não } \\
\text { consegue trazer resultados positivos para os } \\
\text { indicadores básicos de qualidade de vida de forma } \\
\text { satisfatória, o que ocorre graças à eficiência } \\
\text { alocativa dos recursos administrados pela gestão } \\
\text { pública (VLE>0). Aqui, a gestão pública não } \\
\text { consegue evitar perdas líquidas, mas consegue } \\
\text { manter o país afastado da área de pior } \\
\text { desempenho (B3). }\end{array}$ \\
\hline & $\mathrm{A} 2$ & $\begin{array}{c}\text { Present } \\
\text { e }\end{array}$ & Ausente & + & + & - & $\begin{array}{l}\text { Embora com crescimento total acima da média } \\
\text { regional (VLT>0), denotando ganho líquido, a } \\
\text { VLD<0 mostra que o país tem poucas capacidades } \\
\text { de ativação social, o que pode atrapalhar a ação do } \\
\text { poder público, que age de forma eficiente na } \\
\text { alocação de recursos e consegue um desempenho } \\
\text { positivo dos índices de desenvolvimento. }\end{array}$ \\
\hline & & 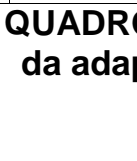 & (3) & & 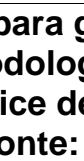 & & $\begin{array}{l}\text { interpretação dos resultados } \\
\text { t-share para os indicadores } \\
\text { ivolvimento humano } \\
\text { a, } 2010 .\end{array}$ \\
\hline
\end{tabular}




\section{Resultados da Aplicação da Metodologia Shift-share}

O quadro 5 apresenta uma síntese dos resultados encontrados dos indicadores educação, longividade e renda. A parte correspondente aos indicadores do IDH, são os elementos utilizados para interpretação do quadro 6, que vai classificar os países de acordo com o quarante correspondente indicados anteriormente no quadro 4.

\begin{tabular}{|c|c|c|c|c|c|c|c|c|c|c|c|c|}
\hline \multirow[b]{3}{*}{ País } & \multicolumn{12}{|c|}{ Resultados do Shift-Share Adaptado de Haddad e Andrade (1989), Silva (2002) e Oliveira (2010) } \\
\hline & \multicolumn{3}{|c|}{ Indicador de Educação } & \multicolumn{3}{|c|}{ Indicador de Longevidade } & \multicolumn{3}{|c|}{ Indicador de Renda } & \multicolumn{3}{|c|}{$\mathrm{IDH}$} \\
\hline & VLT & VLE & VLD & VLT & VLE & VLD & VLT & VLE & VLD & VLT & VLE & VLD \\
\hline Argentina & $-0,060$ & $-0,128$ & 0,067 & 0,151 & 0,158 & $-0,007$ & $-0,020$ & $-0,041$ & 0,021 & 0,070 & $-0,011$ & 0,081 \\
\hline Belize & $-0,184$ & $-0,103$ & $-0,081$ & 0,210 & 0,161 & 0,049 & $-0,191$ & $-0,035$ & $-0,156$ & $-0,165$ & 0,023 & $-0,188$ \\
\hline Bolívia & $-0,296$ & $-0,120$ & $-0,176$ & 0,187 & 0,127 & 0,060 & $-0,149$ & $-0,031$ & $-0,118$ & $-0,258$ & $-0,024$ & $-0,234$ \\
\hline Brasil & $-0,375$ & $-0,120$ & $-0,255$ & 0,199 & 0,149 & 0,050 & $-0,156$ & $-0,038$ & $-0,118$ & $-0,333$ & $-0,010$ & $-0,323$ \\
\hline Chile & $-0,287$ & $-0,124$ & $-0,163$ & 0,207 & 0,168 & 0,039 & $-0,184$ & $-0,041$ & $-0,143$ & $-0,264$ & 0,003 & $-0,267$ \\
\hline Colômbia & $-0,367$ & $-0,119$ & $-0,248$ & 0,200 & 0,150 & 0,050 & $-0,170$ & $-0,037$ & $-0,133$ & $-0,337$ & $-0,006$ & $-0,331$ \\
\hline Costa Rica & $-0,374$ & $-0,119$ & $-0,255$ & 0,208 & 0,169 & 0,039 & $-0,175$ & $-0,039$ & $-0,136$ & $-0,341$ & 0,011 & $-0,352$ \\
\hline Equador & $-0,342$ & $-0,117$ & $-0,225$ & 0,201 & 0,157 & 0,044 & $-0,157$ & $-0,036$ & $-0,121$ & $-0,298$ & 0,004 & $-0,302$ \\
\hline E Salvador & $-0,285$ & $-0,107$ & $-0,178$ & 0,198 & 0,146 & 0,052 & $-0,145$ & $-0,034$ & $-0,111$ & $-0,232$ & 0,005 & $-0,237$ \\
\hline Guatemala & $-0,342$ & $-0,117$ & $-0,225$ & 0,201 & 0,157 & 0,044 & $-0,157$ & $-0,036$ & $-0,121$ & $-0,359$ & 0,013 & $-0,372$ \\
\hline Guiana & $-0,285$ & $-0,107$ & $-0,178$ & 0,198 & 0,146 & 0,052 & $-0,145$ & $-0,034$ & $-0,111$ & $-0,339$ & $-0,024$ & $-0,315$ \\
\hline Haiti & $-0,400$ & $-0,097$ & $-0,303$ & 0,194 & 0,142 & 0,052 & $-0,153$ & $-0,032$ & $-0,121$ & $-0,283$ & 0,014 & $-0,297$ \\
\hline Honduras & $-0,417$ & $-0,127$ & $-0,290$ & 0,197 & 0,130 & 0,067 & $-0,120$ & $-0,028$ & $-0,092$ & $-0,323$ & 0,009 & $-0,332$ \\
\hline México & $-0,366$ & $-0,109$ & $-0,257$ & 0,197 & 0,148 & 0,049 & $-0,154$ & $-0,030$ & $-0,124$ & $-0,291$ & 0,000 & $-0,291$ \\
\hline Nicarágua & $-0,362$ & $-0,102$ & $-0,260$ & 0,206 & 0,150 & 0,056 & $-0,145$ & $-0,027$ & $-0,118$ & $-0,302$ & 0,020 & $-0,322$ \\
\hline Panamá & $-0,290$ & $-0,120$ & $-0,170$ & 0,203 & 0,159 & 0,044 & $-0,152$ & $-0,040$ & $-0,112$ & $-0,238$ & 0,000 & $-0,238$ \\
\hline Paraguai & $-0,316$ & $-0,119$ & $-0,197$ & 0,207 & 0,160 & 0,047 & $-0,182$ & $-0,041$ & $-0,141$ & $-0,325$ & $-0,002$ & $-0,323$ \\
\hline Peru & $-0,362$ & $-0,102$ & $-0,260$ & 0,206 & 0,150 & 0,056 & $-0,145$ & $-0,027$ & $-0,118$ & $-0,234$ & $-0,006$ & $-0,228$ \\
\hline República Dominicana & $-0,290$ & $-0,120$ & $-0,170$ & 0,203 & 0,159 & 0,044 & $-0,152$ & $-0,040$ & $-0,112$ & $-0,313$ & 0,001 & $-0,314$ \\
\hline Uruguai & $-0,374$ & $-0,113$ & $-0,261$ & 0,194 & 0,149 & 0,045 & $-0,133$ & $-0,035$ & $-0,098$ & $-0,290$ & $-0,007$ & $-0,283$ \\
\hline Venezuela & $-0,447$ & $-0,124$ & $-0,323$ & 0,306 & 0,153 & 0,153 & $-0,181$ & $-0,040$ & $-0,141$ & $-0,428$ & $-0,011$ & $-0,417$ \\
\hline
\end{tabular}

Fonte: Elaboração Própria

\section{Quadro 4: Decomposição do crescimento em componentes de variação na america latina-2007 e 2010 Fonte; Elaboração Própria}

O quadro 6 apresenta a classificação dos países por quadrante de acordo com sua variação. Como percebe-se, nenhum dos 21 países conseguiu se enquadrar no primeiro quadrante que é a eficiencia alocativa e capacidade de ativação social positivas além da variação positiva nos demais indicadores de desenvolvimento humano.

A Argentina é o único paíse que se encontra no quadrante II e ainda assim, está em A3, o que significa que possui VLE negativo, porêm, percebe-se que dentre todos os países, Argentina foi a que menos sofreu com a críse em relação ao desenvolvimento social, dessa forma, constatase que a mesma possui capacidade de ativação social (VLD>0) 
intermediária onde a capacidade de ativação social foi capaz de suprimir a ineficiência alocativa (VLE<0) e assim conseguir um maior crescimento na América Latina.

Todos os países correspondentes ao quadrante III, estão em B3, o que significa que possuem uma fraca capacidade de ativação social. Nesses países a sociedade obteve perdas em todos os elementos do IDH, gerados pela fragilidade da internacionalização no processo de crescimento. Aqui, tanto eficiência alocativa quanto ativação social são ausente, o que piora ainda mais o desempenho.

Os países do quadrante IV estão em B2, o que significa que possuem eficiência alocativa presente e VLE $>0$. Esses países apesar das perdas nos indicadores básicos, a gestão pública consegue uma eficiência alocativa positiva, evitando com que o paíse atinja índices piores (B3).

\begin{tabular}{|c|c|c|}
\hline \multicolumn{3}{|c|}{$\begin{array}{l}\text { QUADRO } 6 \text { : RESULTADOS DA METODOLOGIA } \\
\text { SHIFT-SHARE, POR CLASSIFICA ÇÃO E } \\
\text { QUADRANTES NA AMÉRICA LATINA - } 2007 \text { e } 2010\end{array}$} \\
\hline Posição de Quadrante & $\begin{array}{l}\text { Classificaçã } \\
\text { o dos Países }\end{array}$ & País \\
\hline II & A3 & Argentina \\
\hline \multirow{7}{*}{ III } & B3 & Bolívia \\
\hline & B3 & Brasil \\
\hline & B3 & Colômbia \\
\hline & B3 & Guiana \\
\hline & B3 & Peru \\
\hline & B3 & Uruguai \\
\hline & B3 & Venezuela \\
\hline \multirow{13}{*}{ IV } & B2 & Belize \\
\hline & B2 & Chile \\
\hline & B2 & Costa Rica \\
\hline & B2 & Equador \\
\hline & B2 & El Salvador \\
\hline & B2 & Guatemala \\
\hline & B2 & Haiti \\
\hline & B2 & Honduras \\
\hline & B2 & México \\
\hline & B2 & Nicarágua \\
\hline & B2 & Panamá \\
\hline & B2 & Paraguai \\
\hline & B2 & República Doı \\
\hline
\end{tabular}

Fonte: Elaboração Própria 


\section{Considerações Finais}

Percebe-se, que de uma forma ou de outra, todos os países da América Latina foram afetados no desempenho do Índice de Desenvolvimento Humano de 2007 em relação a 2010. O diferencial está no fato de que alguns paíse souberam adminsistra melhor seus recurso para suprir perdas ou simplismente para evitar com que a sociedade sofresse ainda mais com a queda no desempenho do IDH.

A aplicação do método shift-share possibilitou de forma clara, classsificar os 21 países de acordo com seu desempenho, dessa forma, o mesmo se mostra muito útil no que compete a variação de indicadores.

A América Latina, talvez pela sua grande dimensão e diferenças de desempenho econômico e social, seja tão difícil classifica-la através de dados e variáveis, mas, a variação dos indicadores do IDH obtidos através do relatório anual do PNUD de 2007 e 2010, aliada ao shift-share, possibilitou uma maior compreensão de como anda a capacidade dos países de gerir problemas sociais gerados por "disturbios" econômicos.

\section{Referências Bibliográficas}

COSTA, J.S. Compendio de Economia Regional; Cap. 24 A analise de componentes de variação (shift-share). Coimbra: APDR, 2002.

HADDAD, Paulo Roberto. Economia regional: Teorias e métodos de analise. Fortaleza, BND, 1989.

IMEA. A Unila em Construção: um projeto universitário para a América Latina. Foz do Iguaçu: IMEA, 2009.

OLIVEIRA, G.B. O DESENVOLVIMENTO NA REGIÃO METROPOLITANA DE CURITBA: o desempenho dos índices de desenvolvimento econômico. Curitiba: PPGDE, 2010.

PNUD - Programa das Nações Unidas para o Desenvolvimento. Relatório de Desenvolvimento Humano 2007/2008. Combate as alterações climáticas: Solidariedade Humana num mundo dividido. Nova York. EUA .2007.

- Ultrapassar barreiras: Mobilidade e desenvolvimento humao. Nova York. EUA. 2009.

A Verdadeira Riqueza das Nações: Vias parra o Desenvolvimento Humano. Nova York. EUA. 2010.

SIMÕES. Rodrigo Ferreira. Métodos de análise regional e urbana: diagnóstico aplicado ao planejamento. Disponível em: <http://www.cedeplar.ufmg.br/pesquisas/td/TD\%20259.pdf> acesso em 15 de abril de 2012. 
Revista Tecnologia e Sociedade - 1ª Edição, 2012.

ISSN (versão online): 1984-3526

104

SOUZA. Nali de Jesus de. Desenvolvimento Regional .São Paulo: Atlas, 2009.

VASCONCELLOS, António Vale e. Economia Urbana. Porto: Rés, 1984. 